Editorials

\title{
Chronic Visceral Pain and Functional Diseases
}

\author{
${ }^{1,2}$ Victor V. Chaban \\ ${ }^{I}$ Department of Internal Medicine, Charles R. Drew University of Medicine and Science, USA \\ ${ }^{2}$ Department of Medicine, University of California, Los Angeles, USA
}

Article history
Received: 11-06-2017
Revised: 12-06-2017
Accepted: 21-06-2017
Email: victorchaban@cdrewu.edu

\section{Introduction}

The response properties of pelvic extrinsic primary afferent nerves play a significant role in etiology of many functional disorders Hypersensitivity of visceral mechanoreceptors could result from excessive production of modulatory neurotransmitters. In addition to direct stimulation of stretch activated channels on primary afferent neurons located in Dorsal Root Ganglia (DRG), chemicals produced by different target cells that respond to inflammation also modulate the nociception. The incidence of persistent, episodic and chronic visceral pain is more prevalent in females, which suggests hormonal regulation of visceral nociception. Despite extensive research on the properties of pelvic and splanchnic afferent nerves, little is known about the mechanisms underlying normal and pathological signal transduction pathways underlying many functional diseases. Considerable efforts were made by the scientific community and the pharmaceutical industry to develop novel pharmacological treatments aimed at chronic visceral pain, but the traditional approaches to identify and evaluate novel drugs have largely failed to translate into effective therapeutic treatments of functional diseases. Moreover, our recent data that estrogen can gate primary afferent response to modulate nociception support the idea about the involvement of peripheral central system in etiology of a wide range of the functional and inflammatory diseases (Chaban, 2016a).
In general, depending on the channel activation of primary afferent neurons may result in hyperpolarization, depolarization or primarily $\mathrm{Ca} 2+$ influx. The cell bodies of primary visceral spinal afferent neurons located in the lumbosacral (L1-S1) DRGs that transmit information about chemical or mechanical stimulation from the periphery to the spinal cord. Nociceptors are small to medium size DRG neurons whose peripheral processes detect potentially damaging physical and chemical stimuli. Today, multiple lines of evidence suggest that ATP signaling via $\mathrm{P} 2 \mathrm{X}$ receptors and activation of TRPV1 channels contribute to different pain phenotypes, On the other hand, the response of primary afferent neurons may be tonically inhibited by NO produced by peripheral nerve terminals. The peripheral sensitization of nerve fibers is transient depending on the duration of stimuli and presence of visceral inflammation.

\section{Aim of the Study}

To look for the new translational aspects to explain observed comorbidity between different functional disorders and to propose a possible new mechanism for the therapeutic intervention.

\section{Results and Discussion}

Estrogen receptors expressed in visceral nociceptors are associated with functional diseases. Several lines of evidence indicate that $17 \beta$-estradiol (E2) directly 
influence the functions of primary afferent neurons. Both subtypes of Estrogen Receptors (ER $\alpha$ and $E R \beta$ ) are present in DRG neurons including the smalldiameter putative nociceptors. In vitro, ATP-sensitive DRG neurons respond to E2 (Chaban et al., 2011), which correlated well with the idea that visceral afferents are E2 sensitive: (i) Visceral pain is affected by hormonal level in cycling females; (ii) there are sex differences in the prevalence of functional disorders involving the viscera and (iii) putative visceral afferents fit into the population of DRG neurons that are sensitive to E2. These data suggest that in addition to CNS actions, E2 can act in the periphery to modulate nociception (Cho and Chaban, 2012). E2 modulates cellular activity by altering ion channel opening, G-protein signaling and activation of trophic factor-like signal transduction pathways. These effects have been ascribed to membraneassociated receptors. Results from our laboratory and others indicate that E2 acts in DRG neurons to modulate L-type VGCC and through group II metabotropic glutamate receptors (Chaban et al., 2011). E2 has a significant role in modulating visceral sensitivity, indicating that E2 alterations in sensory processing may underlie sex-based differences in functional pain symptoms. Indeed in most clinical studies, women report more severe pain levels, more frequent pain and longer duration of pain than men E2 modulation of nociceptive response depends on the type of pain, its durations and the involvement of other nociceptive-mediated mechanisms.

Primary afferent neurons and viscero-visceral crosssensitization: Emerging model for functional gastrointestinal disorders. Most of the current literature pertains to specific functional syndromes defined by medical subspecialties. These include: IBS (gastroenterology), CPP (gynecology), PBS (urology), fibromyalgia (rheumatology) and others. Many reports described the substantial overlaps between two or more of these syndromes (Chaban, 2016a). Moreover, clinical presentations of functional syndromes lack a specific pathology in the affected organ but may respond to a viscero-visceral cross-sensitization in which increased nociceptive input from an inflamed organ (i.e., uterus) sensitizes neurons that receive convergent input from an unaffected organ (i.e., colon or bladder). The site of visceral cross-sensitivity is unknown.

Recent studies from our laboratory demonstrated that hormonal modulation of visceral inputs of primary afferent nociceptors located in the Dorsal Root Ganglia (DRG) is responsible for changes observed in the perception of pain during the etiology of functional pain syndromes (Chaban, 2014). Individuals suffering from CPP frequently have pain emanating from several visceral organs. Viscero-somatic and viscero-viseral hyperalgesia and allodynia lead to the perception of pain spreading from an initial site to adjacent areas. Patients with CPP may at first have only one source of pain in the pelvis, but numerous mechanisms involving the central and peripheral nervous systems may result in the development of painful sensations in adjacent organs, such as IBS being associated with lower colonic pain (Chaban, 2016b).

\section{Conclusion}

Similar to other chronic diseases, a multi component conceptual model of functional disorders involves physiologic, cognitive and behavior factors will be necessary for developing new therapies. The different systems such as neuroendocrine regulation and autonomic response will affect nociceptive systems. From a public health perspective, a substantial impact on our knowledge of nociceptive functional diseases will help achieve a deeper understanding of data presented in clinical aspects of these symptoms. Only a thorough understanding of the mechanism implicated in these phenomena can truly contribute to the designing of new and more efficient therapies.

\section{Acknowledgement}

Author would like to acknowledge the support from Urban Health Institute of Charles R. Drew University of medicine and Science.

\section{Funding Information}

This work is sponsored by U.S. National Institute of Minority Health and Health Disparities (NIMHD) of National Institute of Health under award numbers: S21 MD 000103.

\section{Ethics}

There are no ethical issues associated with this publication.

\section{References}

Chaban, V., 2014. Chronic pelvic pain: Focus on etiology and modulation. Int. J. Res. Nurs., 5: 1-2.

Chaban, V., 2016a. Irritable bowel syndrome-novel concepts for research and treatment.

Chaban, V., 2016b. Unraveling the Enigma of Visceral Pain. 1st Ed., Nova Publishers, N.Y. pp: 77.

Chaban, V., J. Li, J.S. McDonald, A. Rapkin and P. Micevych, 2011. Estradiol attenuates the adenosine triphosphate-induced increase of intracellular calcium through group II metabotropic glutamate receptors in rat dorsal root ganglion neurons. J. Neurosci. Res., 89: 1707-10. DOI: 10.1002/jnr.22718

Cho, T. and V. Chaban, 2012. Interaction between P2X3 and $\mathrm{ER} \alpha / \mathrm{ER} \beta$ in ATP-mediated calcium signaling in mice sensory neurons. J. Neuroendocrinol., 24: 789-797. DOI: $10.1111 / \mathrm{j} .1365-2826.2011 .02272 . x$ 